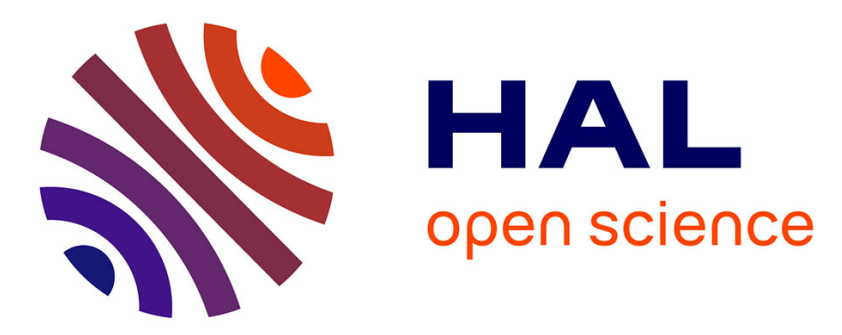

\title{
Characterization of small-scale structures at the magnetopause from ISEE measurements
}

\author{
L. Rezeau, A. Roux, C. T. Russell
}

\section{To cite this version:}

L. Rezeau, A. Roux, C. T. Russell. Characterization of small-scale structures at the magnetopause from ISEE measurements. Journal of Geophysical Research, 1993, 98 (A1), pp.179-186. 10.1029/92JA01668 . hal-00408545

\section{HAL Id: hal-00408545 \\ https://hal.science/hal-00408545}

Submitted on 3 Feb 2021

HAL is a multi-disciplinary open access archive for the deposit and dissemination of scientific research documents, whether they are published or not. The documents may come from teaching and research institutions in France or abroad, or from public or private research centers.
L'archive ouverte pluridisciplinaire HAL, est destinée au dépôt et à la diffusion de documents scientifiques de niveau recherche, publiés ou non, émanant des établissements d'enseignement et de recherche français ou étrangers, des laboratoires publics ou privés. 


\title{
Characterization of Small-Scale Structures at the Magnetopause From ISEE Measurements
}

\author{
L. RezeAu AND A. RouX \\ Centre National d'Etudes des Télécommunications, Centre de Recherches en Physique de l'Environnement, Issy-les-Moulineaux, France \\ C. T. RUSSELL \\ Institute of Geophysics and Planetary Physics, University of California, Los Angeles
}

\begin{abstract}
High-resolution data (covering up to $8 \mathrm{~Hz}$ ) from the flux gate magnetometers on the two ISEE spacecraft are used to analyze ultralow-frequency (ULF) fluctuations observed at the magnetopause and in the adjacent layers. Intersatellite correlations are computed to show that the same structure can be identified in the fluctuations observed on both spacecraft when the interspacecraft distance is small. Then the possibility of deducing the velocity of the structure from two-point measurements is discussed; it is shown that it can be estimated only in certain cases.
\end{abstract}

\section{INTRODUCTION}

Data from several spacecraft have been used to give evidence for the presence of a variety of intense waves at the magnetopause, from OGO 5 to AMPTE (Active Magnetospheric Particle Tracer Explorers) [Holzer et al., 1966; Gurnett et al., 1979; LaBelle and Treumann, 1988]. The importance of these waves is related to the role they could play in the dynamics of the magnetopause and of the surrounding regions. In particular it has been suggested by several authors that intense plasma waves could lead to anomalous cross-B diffusion through the magnetopause [Thorne and Tsurutani, 1991; Gendrin, 1983; Treumann et al., 1991]. The question which is still awaiting an answer is which kind of wave might be the most efficient. The ELF-VLF frequency range was discussed first by Tsurutani and Thorne [1982] and Tsurutani et al. [1989]. The ULF frequency range is also a good candidate, since the level of the turbulence in that range is the highest; this was first suggested by Gendrin [1983]. The presence of intense fluctuations in the ULF range has also been reported in flux transfer events (FTEs) by Anderson et al. [1982] and Farrugia et al. [1988].

While the fact that ULF fluctuations are observed at magnetospheric boundaries is well documented, their nature is still a matter of debate. One of the difficulties in interpreting these waves comes from their fast variations in intensity and from their unsteady spectra that exhibit (or do not exhibit) spectral peaks. Anderson et al. [1991], for instance, have observed these fluctuations on a particular crossing of the magnetopause on AMPTE data. They interpreted them as electromagnetic ion cyclotron waves. So did Farrugia et al. [1988], who observed similar fluctuations over a short period inside a FTE, while during the rest of the FTE no interpretation was possible. Another case study (on ISEE, also for northward

Copyright 1993 by the American Geophysical Union.

Paper number 92JA01668.

0148-0227/93/92JA-01668\$05.00 interplanetary magnetic field), made by Song et al. [1990], concluded that the observed fluctuations are not electromagnetic ion cyclotron waves, since their polarization is not left-handed. Rezeau et al. [1989] used GEOS 2 data to characterize the nature of the ultralow-frequency fluctuations observed at the magnetopause, with five conclusions: (1) The fluctuations are electromagnetic with $\delta \mathrm{E} / \delta \mathrm{B}$ generally of the order of the Alfven velocity. (2) Their spectrum is generally a smoothly decreasing function of the frequency. While always valid in the boundary layer these two conclusions have to be taken with caution inside the magnetopause layer itself because the spectra of the electric component sometimes showed a maximum around the proton gyrofrequency which could indicate the presence of quasi-electrostatic waves; such a conclusion should be confirmed by measurements of more than one component of the electric field [Rezeau, 1988]. (3) The direction of maximum variance of the magnetic components of the fluctuations was found to be always perpendicular to the static magnetic field while the direction of minimum variance was not so well defined. (4) The fluctuations are very intense (up to $10^{2} \mathrm{nT}^{2}$ ). (5) They last short times (typically a few seconds). Thus, while the transverse nature of the fluctuations suggests that they are shear Alfven waves, their large amplitude and the short duration of the bursts are not consistent with a left-handed ion cyclotron plane wave. In a high $\beta$ plasma, the sense of rotation is not an unambiguous means of determining the mode of propagation. Indeed, as shown for instance by Belmont and Rezeau [1987], in a high $\beta$ plasma the ion cyclotron-shear Alfven wave can be righthanded. The short duration and large amplitude of the bursts are more difficult to interpret. Rezeau et al. [1989] suggest two explanations which are not mutually exclusive. First, the observed signature may be that of a cylindrical wave, with an axis more or less aligned with the magnetic field. Second, the nonlinearity of the Alfven wave may lead to its localization in space. Unfortunately there exists no model describing nonlinear Alfven waves developing in a high $\beta$ plasma, as far as we know.

As it is very difficult, with a single spacecraft, to analyze small-scale structures that probably vary in space and time, the 
data from ISEE 1 and 2 offer new possibilities regarding the distinction between spatial and temporal variations. Hence the purpose of this paper is to assess the hypothesis made by Rezeau et al. [1989] that the observed fluctuations correspond to small-scale nonlinear Alfven waves traveling along the magnetopause. To fulfill that goal, correlations between ISEE 1 and 2 will be made to try to identify the same structure on the two spacecraft. This will help to characterize the properties of the structure; an attempt will be made, in particular, to estimate its velocity. This will be done by using data from the flux gate magnetometers on ISEE 1 and 2. After subtraction of a running average over $5 \mathrm{~s}$, the obtained frequency range is 0.2 to $8 \mathrm{~Hz}$. This filtering of the data is applied to remove the large-scale variations of the field.

\section{CORRELATION BETWEEN STRUCTURES OBSERVED ON BOTH SPACECRAFT}

\subsection{Comparison of the Signatures on the Two Spacecraft}

The first case that we consider takes place within a FTE observed at the inner edge of the boundary layer (Figure 1). During the event the large scale variations of the magnetic field are very similar on ISEE 1 and ISEE 2. The spacecraft move from the magnetosphere $(0254: 00)$ to the boundary layer (0255:30); in between, the magnetic field signature and the density enhancement indicate the presence of a FTE [Paschmann et al., 1982]. The internal structure of the FTE

ISEE, 8 November 1977
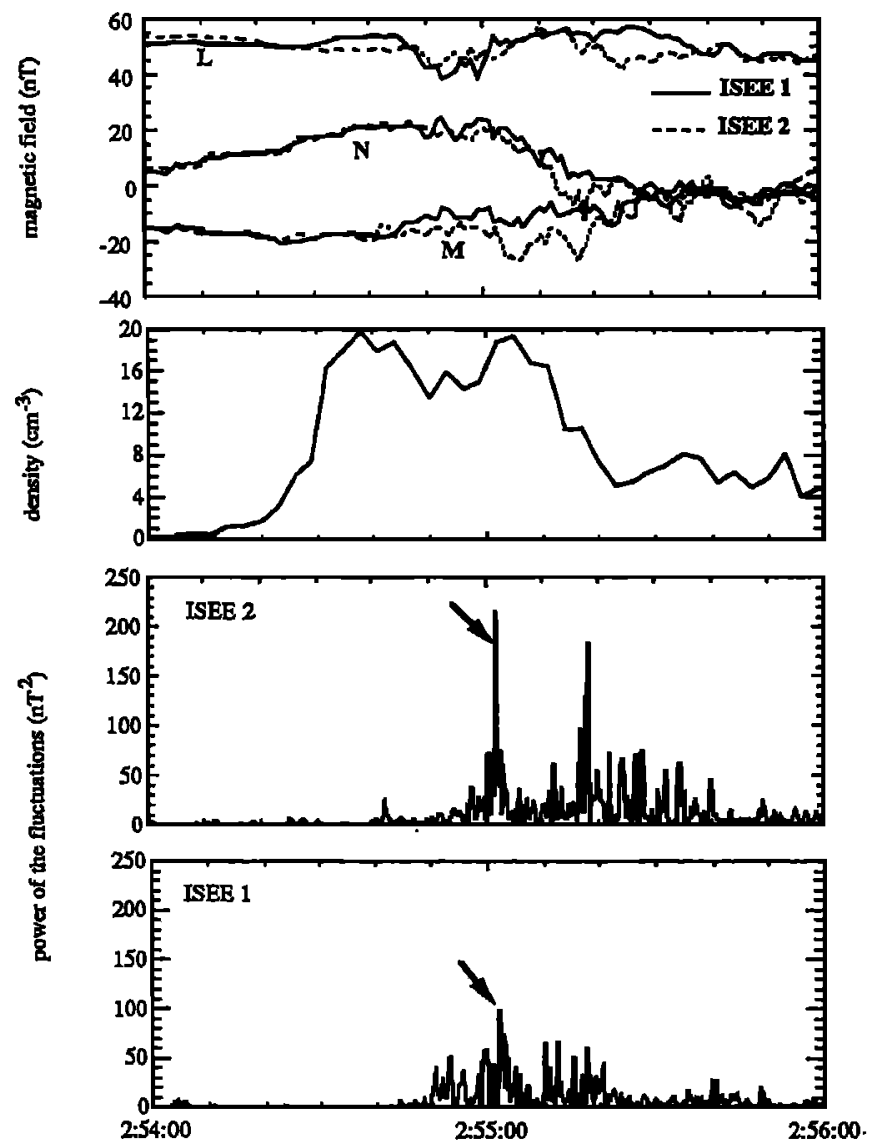

Fig. 1. Variations of magnetic field, density, and power of the fluctuations in the frequency range $0.2-8 \mathrm{~Hz}$. The arrows show the localized structures studied in section 2 .
[Farrugia et al., 1988] can be inferred from the two humps observed in the density: two density gradients (0254:20. 0254:40 and 0255:05-0255:25) are separated by a region with slower variations. In the lower panels, the power of the fluctuations is plotted for the two spacecraft. It is clear that the level of the fluctuations increases in the FTE. A striking feature is also observed: there is a clear asymmetry in the level of the fluctuations, which is much higher on the boundary layer side of the FTE than on its magnetospheric side.

It is also worth noticing that the fluctuations are highly structured; a sharp peak is seen at 0255:02 on ISEE 2 followed by another one, $17 \mathrm{~s}$ later (Figure 1). Since some structures appear approximately at the same time on ISEE 1, one can wonder whether the events observed at the two spacecraft are the magnetic signatures of localized structures passing by the two spacecraft at successive times. The corresponding waveforms are plotted in Figure 2. The first problem to solve is the following: once a given structure is identified on ISEE 2 (at 0255:02), is the maximum observed at approximately the same time on ISEE 1 the signature of the same structure or the signature of another structure? To answer this question, the cross-correlation functions of the three components of the magnetic fluctuations measured at the two spacecraft locations are used. The correlation function is computed in the following way: we consider a time interval (duration $\mathrm{T}$ ) on the spacecraft chosen as a reference, centered on the interesting structure (time $t_{0}$ ). For a given delay $\tau$ the signal on the other spacecraft
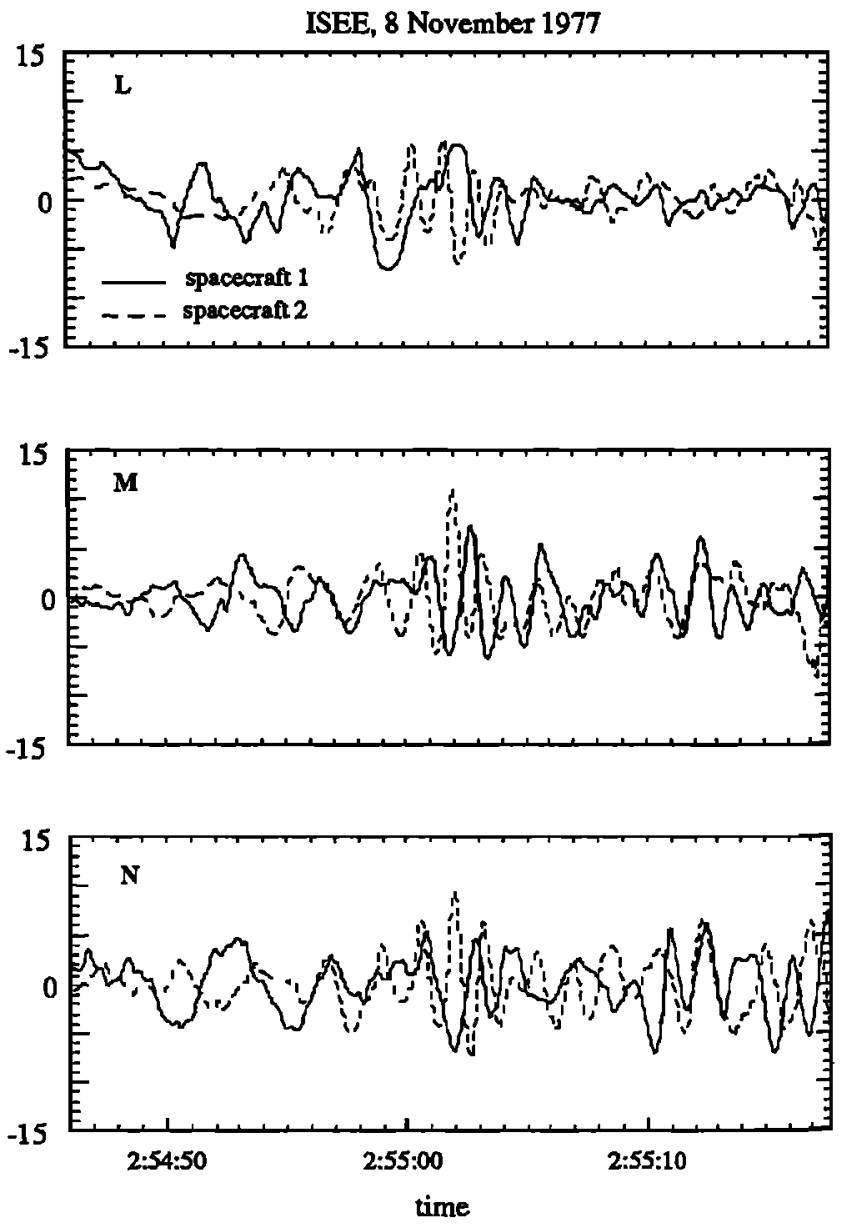

Fig. 2. Waveforms of the fluctuations plotted in the LMN reference frame (nanoteslas). 
is considered in the interval $\left[t_{0}+\tau-T / 2 ; t_{0}+\tau+T / 2\right]$. The correlation function is normalized by the square root of the autocorrelation functions of the two signals, at times $t_{0}$ and $t_{0}+\tau$, respectively. The resuits are plotted in Figure $3 \mathrm{a}$, with spacecraft 2 chosen as the reference, with $t_{0}=0255: 02$ and $\mathrm{T}=8 \mathrm{~s}$. The frame of reference is the magnetopause (LMN) frame. All components show approximately the same behavior: a clear oscillation (with about 1-s period) and about the same value of the correlation function for small positive delays. For the $M$ component a maximum equal to 0.6 is found for a delay of $\approx 2 \mathrm{~s}$. A statistical test has been applied to this value to test its significance. Since the correlation function $p$ does not have a normal distribution, we follow the method described, for instance, by Bendat and Piersol [1986] and calculate the function $w=0.5 \ln [(1+p) /(1-p)]$, which has a normal distribution. The $95 \%$ confidence interval obtained for

ISEE, 8 November 1977

a)

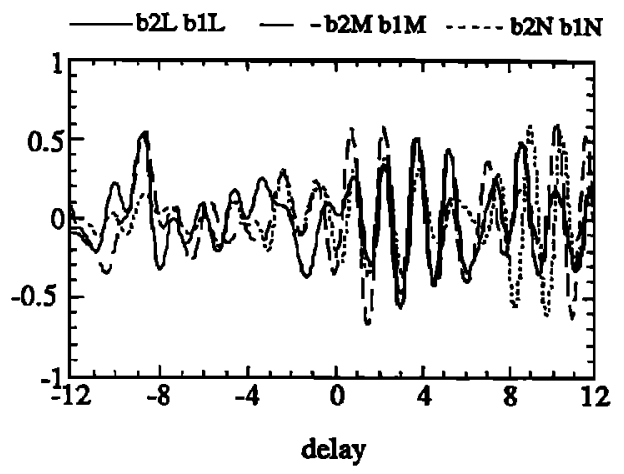

b)

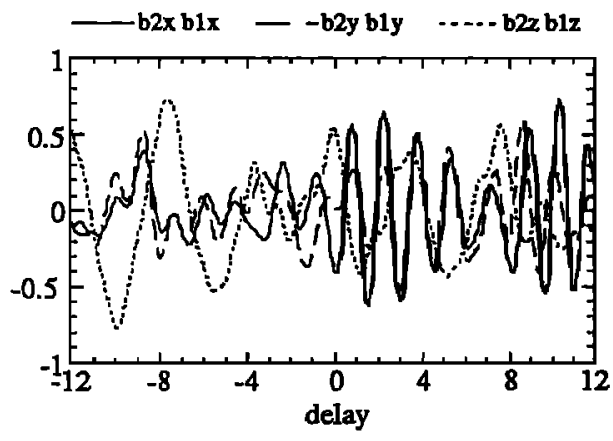

c)

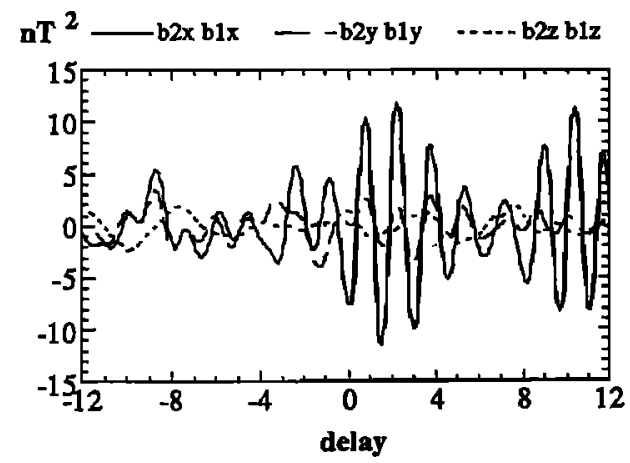

Fig. 3. Correlation functions for the event shown in Figures 1 and 2. The central time is 0255:02 on spacecraft 2, and the data from spacecraft 1 are delayed. (a) The functions are normalized (see text for details) and calculated over 8 in the LMN frame. (b) Same in the minimum variance frame of ISEE 2 $(x, y, z)$. (c) Same as Figure $3 b$, but the functions are not normalized. $p$ is $[0.48 ; 0.70]$, which suggests that the two structures are the same. Of course it is not a $100 \%$ correlation, which would mean that the two spacecraft are crossing exactly identical structures; looking at Figure 1, it is clear that the amplitude of the signature is weaker for ISEE 1 than for ISEE 2. The correlation functions of the other components look similar, but with lower values of the maxima that are obtained for different delays. Another feature to note is that the maximum value of the correlation function is also reached for a larger delay ( $10 \mathrm{~s}$ for the $\mathrm{M}$ component), as though ISEE 1 was crossing a second structure similar to the first one, although possibly oriented differently (the $\mathrm{N}$ components show a strong correlation for the second event, whereas the correlation was weak for the first one).

This correlation study indicates that the two spacecraft are likely to cross successively the same structure, or even a chain of structures (that probably evolved between the two observations).

\subsection{Characterization of the Structure}

This structure will now be characterized on both satellites by using standard methods usually applied on a single spacecraft, and the results obtained for the two spacecraft will be compared. The spectra displayed in Figure 4 are very similar: the general trend is a smooth decreasing law, with an enhancement just below $1 \mathrm{~Hz}$. This enhancement corresponds to the frequency of the oscillations observed on the correlation functions. Looking more precisely, one finds that there is a small shift between the two maxima $(0.6 \mathrm{~Hz}$ on ISEE 1 and $0.8 \mathrm{~Hz}$ on ISEE 2). The spectra plotted in Figure 4 are the sum of the spectral densities of the three components; but it is also necessary to study the components separately, to determine the polarization of the fluctuations. For that purpose, a minimum variance analysis has been performed on the data over $8 \mathrm{~s}$. The main results are summarized in Table 1, where the directions of variance are compared to the direction of the magnetic field and to the direction of the normal $N$. The directions obtained on ISEE 1 are also compared to those obtained on ISEE 2. The polarization is better defined on spacecraft 2 , where the ratio of the minimum to the maximum

\section{ISEE, 8 November 1977}

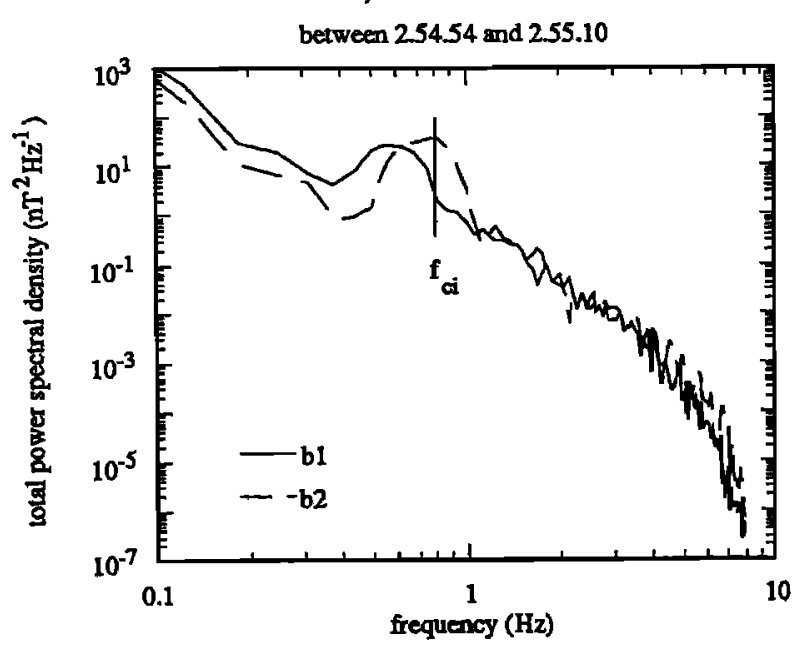

Fig. 4. Comparison of the total spectral densities of the fluctuations on the two spacecraft (sum of the spectra of the three components). 
TABLE 1. Results of Minimum Variance Analysis

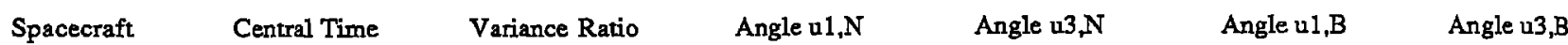

\begin{tabular}{ccccccc}
\hline 1 & 0255.03 & 0.19 & 52 & 42 & 77 & 84 \\
2 & 0255.02 & 0.07 & 46 & 44 & 62 & \\
\hline & $\mathrm{u} 1, \mathrm{u} 1$ & $\mathrm{u} 2, \mathrm{u} 2$ & $\mathrm{u3}, \mathrm{u3}$ & $\mathrm{B}, \mathrm{B}$ & & \\
\hline & 22 & 39 & 35 & 9
\end{tabular}

Here $\mathrm{u} 1, \mathrm{u} 2$, and $\mathrm{u} 3$ are the directions of maximum, intermediate, and minimum variances, $\mathrm{N}$ is the average normal to the magnetopause, and variance ratio is the ratio of minimum to maximum variances. The bottom part of the table shows angles between vectors calculated on both spacecraft.

variance is smaller. The main properties of the minimum variance frames are the following: the directions of maximum $v$ ariance are almost perpendicular to the static magnetic field $\left(77^{\circ}\right.$ and $\left.88^{\circ}\right)$, and the direction of minimum variance is not parallel to the magnetic field nor to the magnetopause normal. This confirms the results obtained on board GEOS 2, where the most prominent characteristic was also a direction of maximum variance perpendicular to the static magnetic field. Furthermore, the variance frames are similar on both spacecraft. To compare more precisely the polarizations, the hodograms are plotted in the plane of maximum variance obtained on spacecraft 2 (Figure 5). The polarizations look similar: elliptic polarization, with the same sense of rotation (right-hand with respect to the minimum variance direction and with respect to the magnetic field). This right-hand polarization is at variance with the findings of Farrugia et al. [1988]. On the hodogram of spacecraft 2, it is clear that the amplitude of the field first increases and then decreases; this can also be seen in the waveforms of the fluctuations plotted in the spacecraft 2 variance frame (Figure 6). All these observations confirm that the same structure is likely to be observed on both spacecraft. The next question is: what else can be deduced from these two-point measurements, once we know that it is the same structure? In particular, is it possible to estimate the velocity of the structure?

\subsection{Estimate of the Velocity of the Structure}

To help the intepretation, a simple example is considered: a thin cylindrical current tube moving by the spacecraft at a constant velocity. Minimum variance analysis applied to the magnetic signature of a current tube gives the direction of the axis of the structure (minimum variance direction) and the direction of its velocity relative to the spacecraft (intermediate variance direction) [Rezeau et al., 1989]. Thus if the variance frames obtained on the two spacecraft are parallel, the common direction of intermediate variance is the direction of the velocity, or at least the direction of its projection on the plane perpendicular to the current. To estimate the magnitude of the velocity, one can use the correlation function. In Figure 7a the magnetic field signature of a thin cylindrical current tube, moving at $100 \mathrm{~km} \mathrm{~s}^{-1}$, is plotted in the minimum variance frame ( $\mathrm{z}$ is parallel to the direction of the current, and $\mathrm{x}$ to the direction of the velocity). The structure is seen by two spacecraft $255 \mathrm{~km}$ apart, and the correlations between the waveforms are plotted in Figure $7 \mathrm{~b}$. The correlation functions show a clear extremum (almost equal to 1) for a delay of $-2.5 \mathrm{~s}$, which is exactly equal to the ratio between the projection of the spacecraft separation in the direction of the velocity $(250 \mathrm{~km})$, and the velocity $\left(100 \mathrm{~km} \mathrm{~s}^{-1}\right)$. This result shows that it is possible to estimate at least one component of the velocity of a moving current tube (the component which is perpendicular to the symmetry axis of the structure).

ISEE, 8 November 1977

magnetic fluctuations between 2:55:00 and 2:55:04
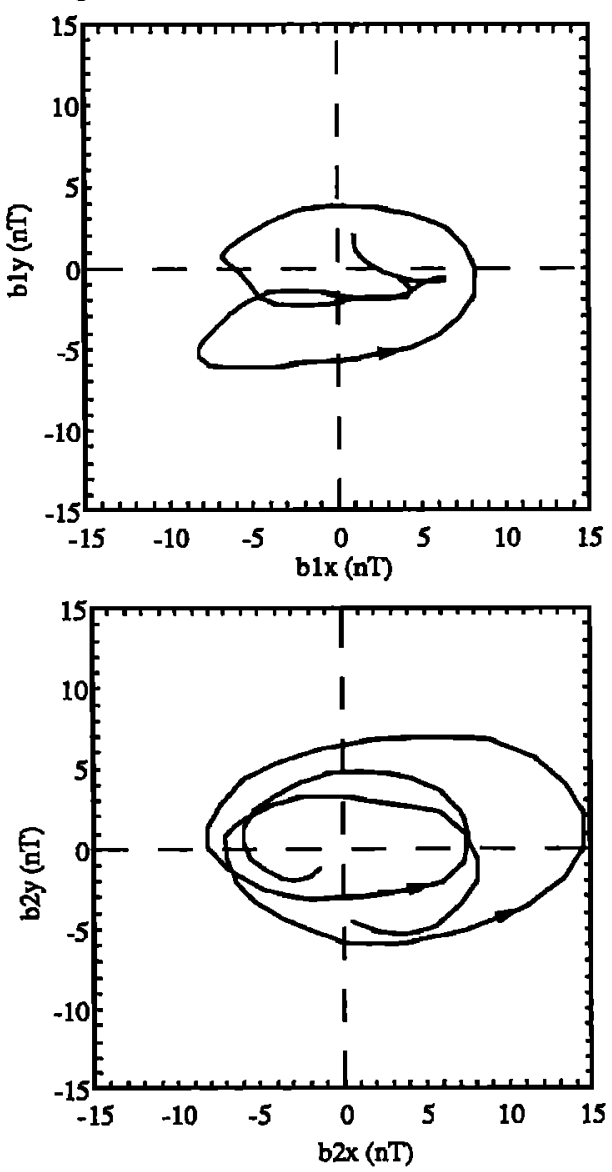

Fig. 5. Hodograms of the fluctuations in the plane defined by maximum and intermediate variance directions (calculated on ISEE 2). The sense of rotation is indicated by arrows. 

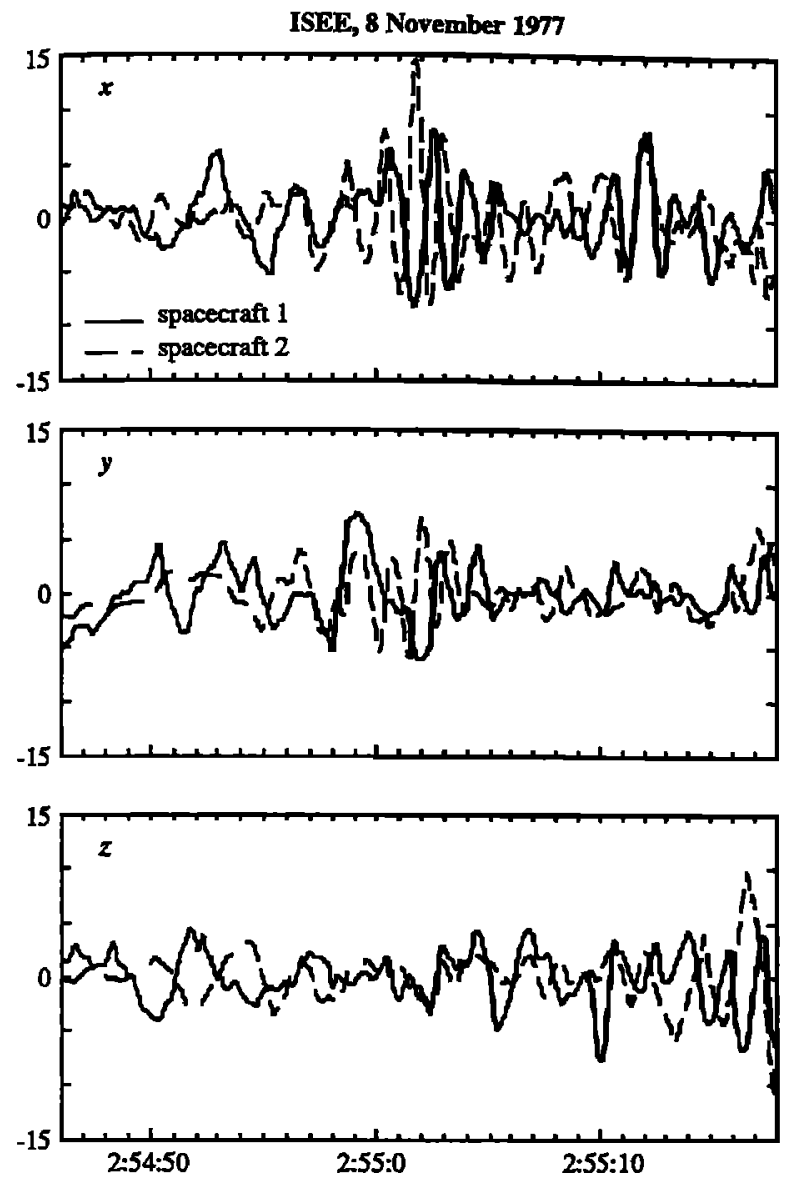

Fig. 6. Waveforms of the fluctuations plotted in the minimum variance frame of ISEE 2 (nanoteslas), for the same time period as Figure 2.

Let us apply the same method to the case studied previously from ISEE data. The minimum variance analysis gives the direction of intermediate variance: its components are $(-0.996$, $0.051,0.076)$ in the LMN frame. One possible hypothesis to be checked is whether the structure is being carried by the plasma; to assess this hypothesis, we must compare this velocity to the projection of the ion velocity on the plane perpendicular to the axis of the structure (actually, it is the only component of the flow velocity that can be measured if the structure is assumed to have a cylindrical symmetry). In GSE coordinates the ion velocity direction was found to be approximately characterized by $\Lambda=-45^{\circ}$ and $\Phi=-90^{\circ}$ at that time [Paschmann et al., 1978]. Once this velocity direction is projected in the LMN frame, we get $(-0.864,-0.355,-0.357)$. This direction is compared first to the minimum variance direction; since they are found to be almost perpendicular, the velocity of the structure can be compared to the velocity of the plasma (both of them being in the maximum variance plane). The angle between them is equal to $35^{\circ}$, and consequently the two velocity vectors are not too far from being parallel.

Let us now compute the correlation functions in the variance frame (Figure 3b). The method is the same as for Figure 3a, but the results are quite different. For the $x$ component, the same features as before are found again, but the behavior of the other components is quite different: the same oscillation exists in the $y$ component, but weaker; for the $\mathrm{z}$ component this fast oscillation is absent, but another, slower one has appeared.
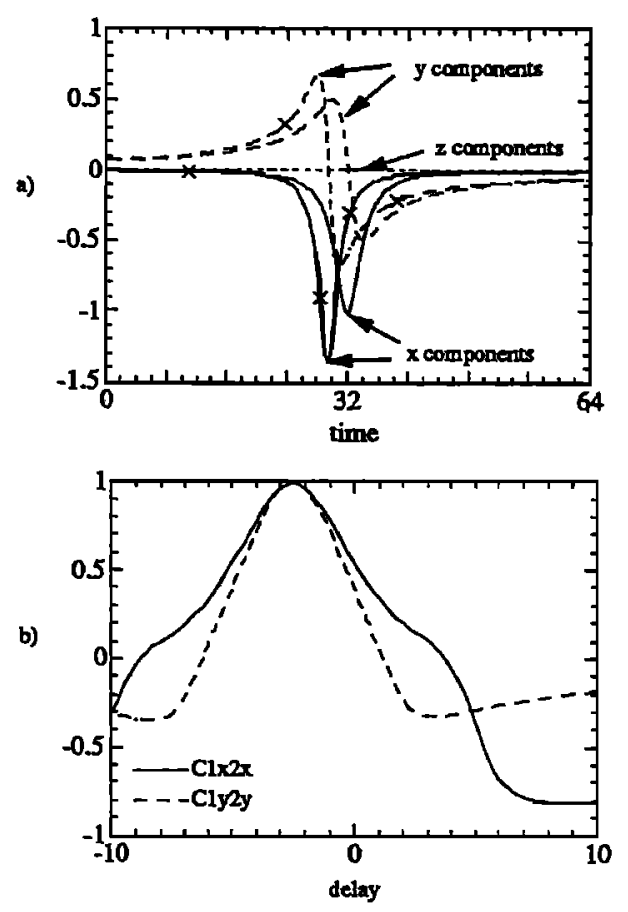

Fig. 7. (a) Magnetic signatures of a current tube passing by two spacecraft. The crosses indicate the components of the field on spacecraft 2 . The frame of reference is the minimum variance frame (or, equivalently, $x$ along the velocity of the structure, and $z$ parallel to the direction of the current). (b) The correlation functions between the signatures shown in Figure 7 a.

For the $x$ component, the maxima are similar: 0.65 and 0.7 for delays of 2 and $10 \mathrm{~s}$. From the smallest delay and from the interspacecraft distance along the direction of intermediate variance $(110 \mathrm{~km})$, the magnitude of the velocity is deduced: about $55 \mathrm{~km} \mathrm{~s}^{-1}$. The plasma instrument measures at that time a velocity of $65 \mathrm{~km} \mathrm{~s}^{-1}$. Considering the lack of precision of our estimates, these results are consistent with the magnetic structure being carried by the plasma flow, along the magnetopause.

\subsection{Interpretation}

The present study, which is based on dual-spacecraft data and comparison with a model, confirms the conclusion drawn by Rezeau et al. [1989] on the basis of GEOS 2 data. Shortduration bursts in ULF fluctuations observed at, or close to, the magnetopause are the magnetic signatures of small-scale structures passing by the spacecraft. The maximum variance of the observed fluctuations is found to be perpendicular to the magnetic field. This, together with the observations carried out on magnetic and electric components of the fluctuations observed in the same region (magnetopause and boundary layer) by GEOS 2 [Rezeau et al., 1989], leads to the conclusion that these fluctuations are Alfvenic (of shear Alfven type). This spatial confinement of the fluctuations is likely to be due to nonlinear effects: a hypothesis that is supported by their large amplitudes $(\delta B / B \approx 0.25)$. Several studies have been devoted to nonlinear Alfvenic solitons [e.g., Sheerin and Ong, 1980]. An interesting model has been elaborated by Chmyrev et al. [1988] for ionospheric Alfvenic structures: they demonstrate that a drift instability associated with a density 
gradient can generate chains of Alfvenic vortices. In our case, this idea is interesting since (1) a density gradient is present at the inner edge of the boundary layer, and (2) the observed magnetic signatures are consistent with a chain of structures (in Figure 1, the structure which has been studied above is followed by another, similar one; the correlation function does show two maxima with similar values as shown in Figure 3). The problem with the above interpretation is that the plasma is assumed to have a small $\beta$ value, whereas it is clear that $\beta$ is usually of the order of 1 at the magnetopause [Sonnerup et al., 1981]; by now, no equivalent model exists for high beta plasma. It can be checked that in the case we have studied, the polarization of the fluctuations (right-hand polarization) is consistent with the polarization obtained in the frame of a linear theory when $\beta \approx 0.5$ [Belmont and Rezeau, 1987]. Then, since $\beta$ is large, the kinetic aspect of the Alfven wave is more likely to be due to temperature than to the electron inertia. The difficulty in establishing a proper model of kinetic Alfven waves comes first from the high $\beta$ and second from the determination of the free energy source that leads to the generation of these structures. The density gradient is not the only possible instability factor; actually, the fluctuations are observed also on magnetopause crossings, when there is a magnetic field rotation and not always a density gradient (when the density gradient is distinct from the magnetic discontinuity). Thus two different mechanisms might take place to generate these structures.

\section{Discussion}

It has been shown that it is possible to identify the same small-scale structure on two spacecraft $350 \mathrm{~km}$ apart and to infer some of its properties from these dual measurements. Let us now assess the generality of the methods that have been applied to this particular event.

\subsection{Suitability of the Correlation to Identify a Structure}

The above structure has been identified by comparing signatures on both spacecraft and especially by computing normalized correlation functions. It is not clear that the correlation between the two signatures of a given structure should always be conclusive. For instance, if we consider the signature of a stationary localized structure, there might be an effect of "perspective": for a current tube signature, the typical width of the magnetic signature is inversely proportional to the distance of closest approach [Rezeau et al., 1990]. Thus if the distances of closest approach are different for the two spacecraft, this is equivalent to correlating functions with different widths (corresponding to different frequencies); consequently, the correlation function is not necessarily close to 1, although the "object" is the same. This effect might explain why in the case studied in section 2, slightly different frequencies, 0.6 and $0.8 \mathrm{~Hz}$, were obtained from the Fourier analysis of data collected at the two spacecraft locations. This would also explain why the maximum of the normalized correlation function only reached 0.6 .

\subsection{Influence of the Width of the Spectra}

The spectra of the fluctuations are very broad, and all frequencies are present most of the time (Figure 4). A given frequency is likely to be present in the signal, on both spacecraft, even with a low intensity. Thus the efficiency of the correlation function, normalized by the power of the signal, to select specific frequencies (present at the two spacecraft locations) depends strongly on the actual intensities. A means of selecting interesting events (intense together with correlated) could be to use unnormalized correlation functions; yet in this case, the maximum value of the function is more difficult to interpret. Figure $3 c$ shows the unnormalized correlation functions for the case of ISEE data on November 8, 1977, around 0255:02 (same as Figure 3b). Two of the features appearing in the normalized correlation functions do not show up in the unnormalized correlation functions: the local maxima observed for a delay of $-9 \mathrm{~s}$ have a lower amplitude, and the rather high values obtained for the $\mathrm{z}$ component turn out to have no significance since this component has no power.

Another example is studied in Figures 8 and 9. It is located again at the inner edge of the boundary layer which is crossed from boundary layer to magnetosphere $2 \mathrm{~min}$ after the crossing studied previously. A maximum in the power on board ISEE 1 is observed at 0257:24. Looking at the correlation functions, this event seems to be correlated mainly with the event observed $9 \mathrm{~s}$ before on ISEE 2. The confidence interval for the normalized correlation function ( $\mathrm{L}$ component) is $[0.58$; 0.77]. The minimum variance frames calculated for these two events are similar (less than $20^{\circ}$ of difference). Thus in this case again, the same structure is likely to be observed by both spacecraft. It can be noticed also that, as in the case previously studied, other maxima can be observed in the correlation function (for a delay of $-3 \mathrm{~s}$, for instance); consequently it is likely that a chain of correlated structures is crossed by the spacecraft.

ISEE, 8 November 1977
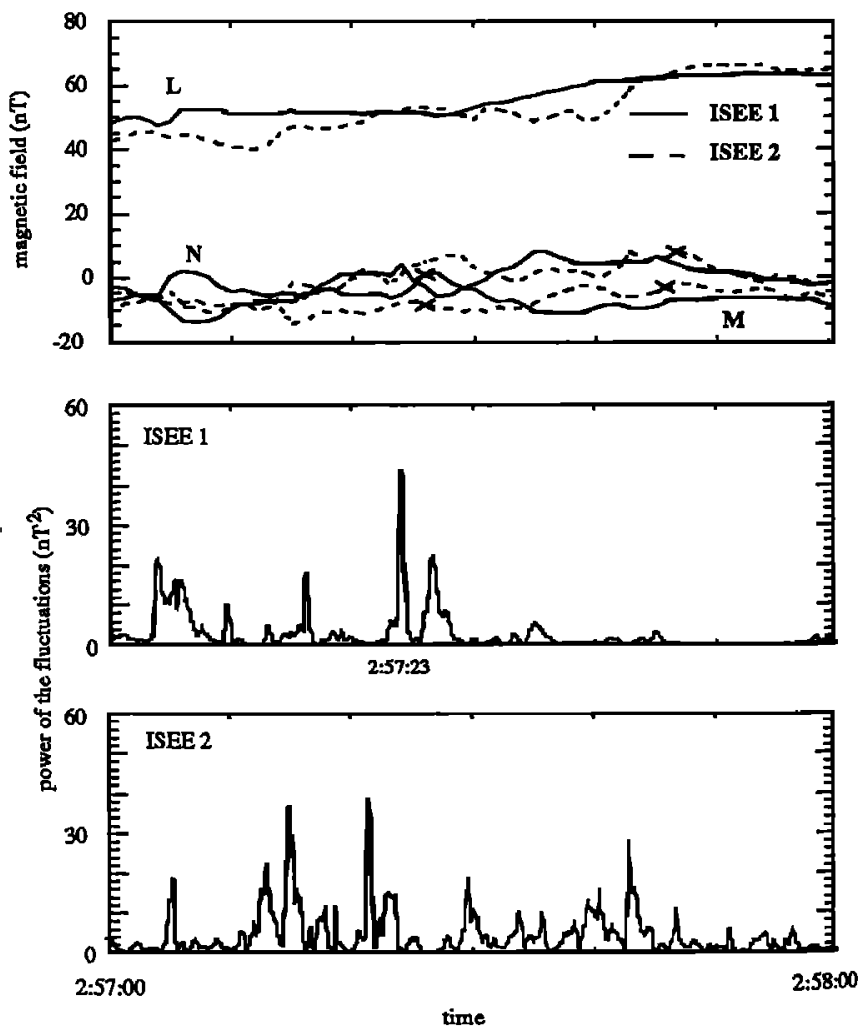

Fig. 8. Variations of magnetic field (top panel), and power of the fluctuations (lower panels). 
8 November 1977

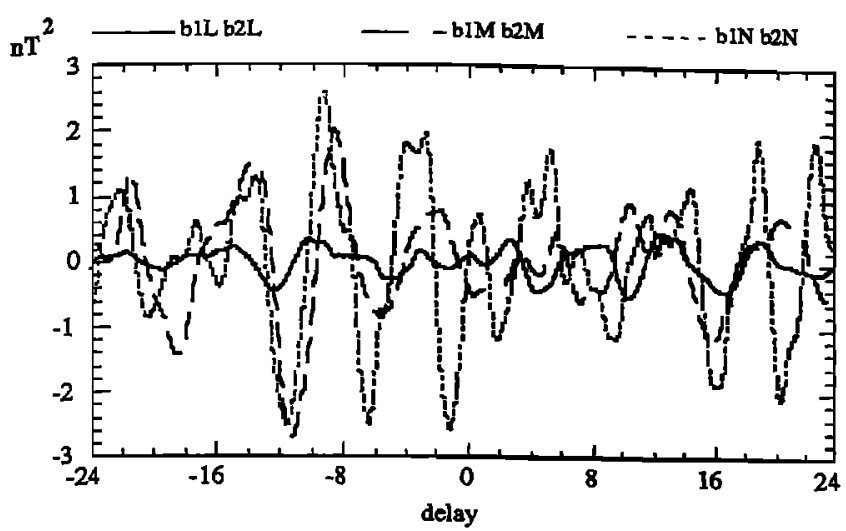

Fig. 9. Unnormalized correlation functions for the event identified at 0257:23 on ISEE 1 (Figure 8).

The consequence of the two points discussed above is that in some cases we can efficiently identify the same structure passing by the two spacecraft from the use of the correlation functions, especially when the two spacecraft are located at the same distance from a given structure, which means that the interspacecraft distance is small. In a more general context, another method should be found to identify a structure when the apparent frequencies are different on the two spacecraft.

\subsection{Improvement of the Model}

The velocity of the structure has been estimated by combining minimum variance analysis with interspacecraft correlation functions. This was tested on a current tube model. Let us now briefly discuss a more realistic model which takes into account the fact that the fluctuations are Alfvenic and localized but does not assume stationarity in the frame of the structure. Rezeau et al. [1989] have discussed the case of a cylindrical Alfven wave, in the linear approximation for a cold plasma. In the case of hot plasma, the solution is the product of Bessel functions of the radial distance (order 1 and 0 ) by a sinusoidal function describing the parallel propagation. An example of the signature of such an Alfvenic structure is shown in Figure 10: it is assumed to be developing under the same conditions as on November 8, 1977, at 0255. It is also assumed to be moving at the plasma velocity past the two ISEE spacecraft. Two parameters are free in the model: the parallel phase velocity and the parallel wavelength; the perpendicular extension is then set by the model. The parallel phase velocity is chosen equal to $367 \mathrm{~km} \mathrm{~s}^{-1}$, and the wave length is adapted to obtain an oscillation at a frequency comparable to the experimental one. The comparison between Figure 6 and Figure 10 shows that the cylindrical Alfven wave is a reasonable model to fit ULF fluctuations: the model field has components in all directions (parallel and perpendicular to the magnetic field), and the same kind of apparent localization is observed. The minimum variance analysis applied to these components allows us to find the direction of minimum variance of the structure (at $1^{\circ}$ of the direction of the static magnetic field) and the direction of the velocity. But the maxima of the correlation functions do not give the exact magnitude of the velocity (the delay is $2 \mathrm{~s}$ instead of $6 \mathrm{~s}$ ). The reason for this indeterminacy could be that the structure is not stationary.
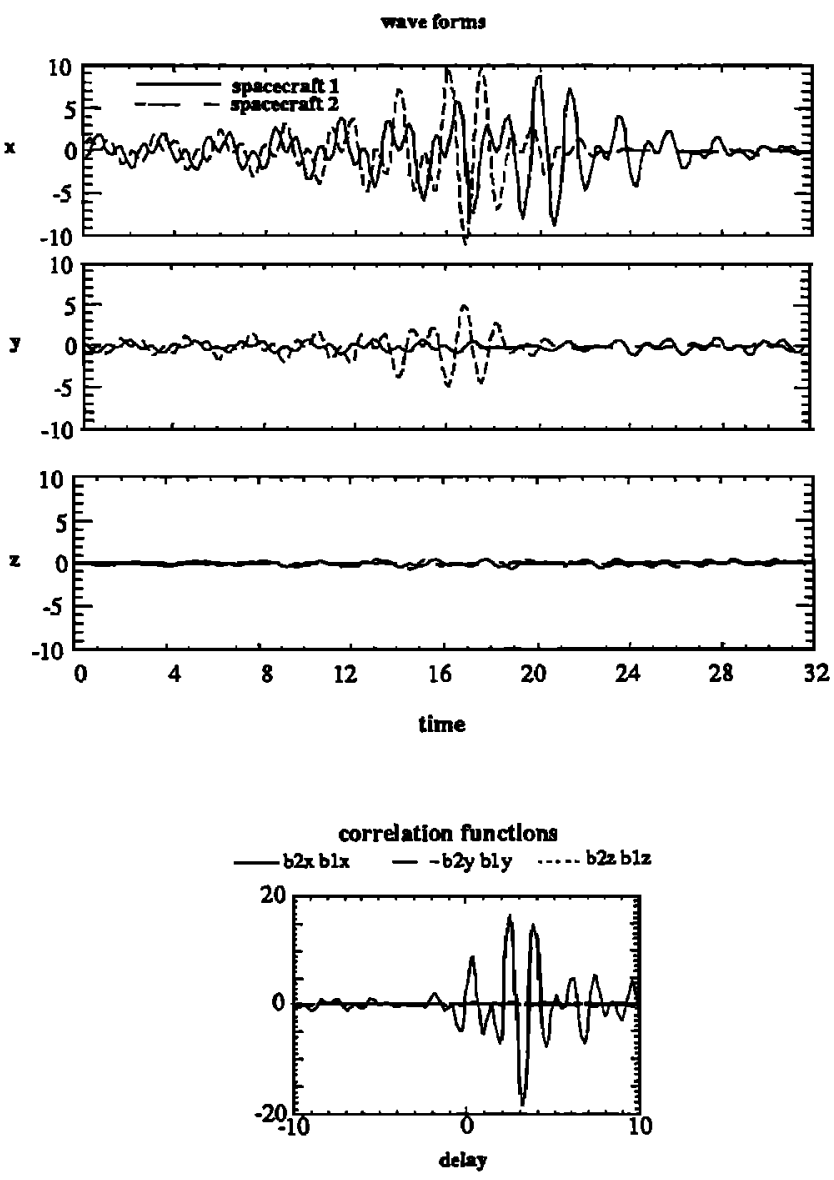

Fig. 10. Magnetic signatures of a linear cylindrical kinetic Alfven wave seen by two spacecraft. The frame of reference is the minimum variance frame ( $\mathrm{z}$ is parallel to the symmetry axis of the structure, and $z$ to the velocity). Below are the correlation functions of the three components (normalized). The vertical units are arbitrary.

\subsection{Discussion of the Model}

The cylindrical Alfven model can provide a reasonable fit, but it is difficult to understand why such a localized structure should develop at some places rather than at others. Given the large amplitude of the structure $(\delta \mathrm{B} / \mathrm{B} \approx 0.25)$, it is tempting to think that the spatial confinement of the structure results from a filamentation of the plasma due to the nonlinearity. Then the spatial extension would result from the balance between nonlinear effects and dispersion. Nonlinear models proposed by Sheerin and Ong [1980] or Chmyrev et al. [1988] give evidence for cylindrical localized structures, or chains of structures localized along the generating discontinuity which would fit very well with our findings. As discussed above, the assumption made by these authors that $\beta$ is much smaller than 1 is not valid in the present context.

\section{CONCLUSION}

ULF fluctuations appear as short-duration (a few seconds), intense $(\delta B / B$ up to 0.25 ) bursts. Single-point measurements do not allow us to assess whether this short duration results from temporal or spatial variations. By using the two ISEE spacecraft, $350 \mathrm{~km}$ apart, we were able to identify the same structure at the two locations, with a delay of about $2 \mathrm{~s}$ between 
the two signatures. This suggests that the short duration of the ULF bursts primarily results from the fast motion of a spatially localized structure moving by the spacecraft. The delay between the two magnetic signatures is consistent with the structure moving at about $55 \mathrm{~km} \mathrm{~s}^{-1}$, which is of the same order and is in the same direction as the measured flow velocity along the magnetopause at that time. Given the variability of the flow, the above findings should only be considered as an indication and not as a definitive proof.

Comparison between the magnetic signatures of the observed structures and that of a model cylindrical structure has been carried out. It shows a good agreement, thereby suggesting that ISEE 1 and 2 were observing cylindrical Alfvenic structures carried by the flowing plasma.

In the cases studied in this paper the interspacecraft distance was less than $500 \mathrm{~km}$, and still there were large differences in the observations of the magnetopause and adjacent regions by the two spacecraft. This means that the boundaries have time to evolve and to move between the observations at the two spacecraft locations. To be able to study a small-scale structure from its signatures on both spacecraft, the first condition is that the boundary on which it is observed is similar at the two locations; otherwise it would not be possible to recognize the structure. The conclusions which come out of this study for future multiple-spacecraft studies of the boundaries are that (1) correlations are only possible for short interspacecraft distances and (2) it is necessary to develop new tools to make the best use of multiple-spacecraft data in cases. where the correlation functions do not provide unambiguous results.

Acknowledgments. We are grateful to J. T. Gosling, S. J. Bame and G. Paschmann for freely providing the FPE data used in this study.

The Editor thanks B. T. Tsurutani and another referee for their assistance in evaluating this paper.

\section{REFERENCES}

Anderson, B. J., S. A. Fuselier, and D. Murr, Electromagnetic ion cyclotron waves observed in the plasma depletion layer, Geophys. Res. Lett., 18, 1955-1958, 1991.

Andérson, R. R., C. C. Harvey, M. M. Hoppe, B. T. Tsurutani, T. E. Eastman, and J. Etcheto, Plasma waves near the magnetopause, J. Geophys. Res., 87, 2087-2107, 1982.

Belmont, G., and L. Rezeau, Finite Larmor radius effects: The two-fluid approach, Ann. Geophys., Ser. A, 5, 59-70, 1987.

Bendat, J. S., and A. G. Piersol, Random Data: Analysis and measurements procedures, John Wiley, New York, 1986.

Chmyrev, V. M., S. V. Bilichenko, O. A. Pokhotelov, V. A. Marchenko, V. I. Lazarev, A. V. Streltsov, and L. Stenflo, Alfven vortices and related phenomena in the ionosphere and the magnetosphere, Phys. Scr., 38, 841-854, 1988.

Farrugia, C. J., R. P. Rijnbeek, M. A. Saunders, D. J. Southwood, D. J. Rodgers, M. F. Smith, C. P. Chaloner, D. S. Hall, P. J. Christiansen, and L. J. C. Woolliscroft, A multi-instrument study of flux transfer event structure, J. Geophys. Res., 93, 14,465-14,477; 1988.

Gendrin, R., Magnetic turbulence and diffusion processes in the magnetopause boundary layer, Geophys. Res. Lett., 10, 769-771, 1983.
Gumetr, D. A., R. R. Anderson, B. T. Tsurutani, E. J. Smith, G. Paschmann, G. Haerendel, S. J. Bame, and C. T. Russell, Plasma wave turbulence at the magnetopause: Observations from ISEE 1 and 2, J. Geophys. Res., 84, 7043-7058, 1979.

Holzer, R. E., M. G. McLeod, and E. J. Smith, Preliminary results from the OGO 1 search coil magnetometer: Boundary positions and magnetic noise spectra, J. Geophys. Res., 71, 1481-1486, 1966.

LaBelle, J., and R. A. Treumann, Plasma waves at the dayside magnetopause, Space Sci. Rev., 47, 175-202, 1988.

Paschmann, G., N. Sckopke, G. Haerendel, J. Papamastorakis, S. J. Bame, J. R. Asbridge, J. T. Gosling, E. W. Hones, Jr., and E. R. Tech, ISEE plasma observations near the subsolar magnetopause, Space Sci. Rev., 22, 717, 1978.

Paschmann, G., G. Haerendel, I. Papamastorakis, N. Sckopke, S. J. Bame, J. T. Gosling, and C. T. Russell, Plasma and magnetic field characteristics of magnetic flux transfer events, $J$. Geophys. Res., 87, 2159-2168, 1982.

Rezeau L., Turbulence des ondes d'Alfven au voisinage de la magnétopause terrestre, thesis, Paris 6 Univ., 1988.

Rezeau, L., A. Morane, S. Perraut, A. Roux, and R. Schmidt, Characterization of Alfvenic turbulence in the magnetopause boundary layer, J. Geophys. Res., 94, 101-110, 1989.

Rezeau, L., A. Roux, and N. Comilleau-Wehrlin, Multipoint study of small scale structures at the magnetopause, in Proceedings of Intemational Workshop on Space Plasma Physics Investigations by Cluster and Regatta, Graz, February 1990, Eur. Space Agency Spec. Publ., ESA SP-306, 103-108, 1990.

Sheerin, J. P., and R. S. B. Ong, Solitary Alfven waveguide structures in a magnetized plasma, J. Plasma Phys., 24, 157-162, 1980.

Song, P., R.,C. Elphic, C. T. Russell, J. T. Gosling, and C. A. Cattell, Structure and properties of the subsolar magnetopause for northward IMF: ISEE observations, J. Geophys. Res., 95, 6375-6387, 1990.

Sonnerup, B. U. Ö., G. Paschmann, I. Papamastorakis, N. Sckopke, G. Haerendel, S. J. Bame, J. R. Asbridge, J. T. Gosling, and C. T. Russell, Evidence for magnetic field reconnection at the Earth magnetopause, J. Geophys. Res., 86, 10.049-10.067, 1981.

Thome, R. M., and B. T. Tsurutani, Wave particles interactions in the magnetopause boundary layer, in Physics of Space Plasmas, SPI Conf. Proc. Reprint Ser., vol. 10, edited by T. Chang, G. B. Crew, and J. R. Jasperse, p. 1-32, Scientific, Cambridge, Mass., 1991.

Treumann, R. A., J. LaBelle, and R. Pottelette, Plasma diffusion at the magnetopause: The case of lower hybrid drift waves, J. Geophys. Res., 96, 16,009-16,013, 1991.

Tsurutani, B. T., and R. M. Thome, Diffusion processes in the magnetopause boundary layer, Geophys. Res. Lett., 9, 1247-1250, 1982.

Tsurutani, B. T., A. L. Brinca, E. J. Smith, R. T. Okida, R. R. Anderson, and T. E. Eastman, A statistical study of ELF-VLF plasma waves at the magnetopause, J. Geophys. Res., 94, 1270-1280, 1989.

L. Rezeau and A. Roux, CNET-CNRS, 38-40 rue du Général Leclerc, F-92131 Issy-les-Moulineaux, France.

C. T. Russell, IGPP, University of Califomia, Los Angeles, CA 90024.

(Received January 6, 1992;

revised July 3, 1992;

accepted July 3, 1992.) 